\title{
Pseudo-Galeno, Praesagitio omnino vera expertaque: primera edición crítica
}

\author{
Elsa García Novo ${ }^{1}$
}

Recibido: 28 de Noviembre de 2020 / Aceptado: 7 de Enero de 2021

Resumen. Se trata de una composición tardía sobre el pronóstico, hecha a partir de textos de Hipócrates y Galeno, tomados en su mayoría de compiladores. Se encuentra en tres manuscritos del siglo XV, Parisinus gr. 2269, Mutinensis gr. a. U. 9. 04 (61 Puntoni), y Palatinus gr. 143. No existen traducciones medievales. La primera edición es de René Chartier en 1639, hecha sobre el Parisino. Giorgio Valla tradujo el texto del Mutinense (1498). Se recogen los loci similes, así como rasgos de la transmisión y de la lengua. Esta es la primera edición crítica del tratado, a la que se añade stemma codicum y traducción al castellano.

Palabras clave: Galeno, Pseudo-Galeno, pronóstico, fiebres, sudoración, Giorgio Valla, René Charterius.

\section{[en] Pseudo-Galen, Praesagitio omnino vera expertaque: first critical edition}

Abstract. We deal with a late composition on prognosis. The texts are taken nearly verbatim from compilers of Hippocrates and Galen. There are three copies of the treatise, in the manuscripts Parisinus gr. 2269, Mutinensis gr. a. U. 9. 04 (61 Puntoni), and Palatinus gr. 143, all of them dating from the XVth century. There are no medieval translations. The first edition is by René Chartier (1639), who read the Parisinus. Giorgio Valla translated the text of the Mutinensis (1498). This is the first critical edition of the treatise. Transmission features, language study, loci similes, a stemma codicum and a translation into Spanish are added.

Keywords: Galen, Pseudo-Galen, prognosis, prognosis by means of fevers, prognosis by means of sweating, Giorgio Valla, René Charterius.

Sumario. 1. Introducción. 2. Traducciones medievales. 3. Los códices griegos y sus relaciones. 4. Stemma codicum. 5. Las ediciones del texto griego. 6. Las traducciones latinas. 7. Composición del tratado y fuentes: los loci similes. 8. Posible datación. 9. La lengua del tratado. 10. Edición del texto. 11. Traducción.

Cómo citar: Garcia Novo, E. (2021), Pseudo-Galeno, Praesagitio omnino vera expertaque: primera edición crítica, en Cuadernos de Filología Clásica. Estudios griegos e indoeuropeos 31, 107-122.

1 Universidad Complutense de Madrid, Departamento de Filología Clásica.
egarcian@ucm.es 


\section{Introducción}

Praesagitio omnino vera expertaque es una composición muy tardía hecha por un imitador de Galeno, que ha reunido unas cuantas señales de muerte próxima, extraídas de Hipócrates, Galeno y Aecio Amideno, así como de compilaciones tardías sobre las fiebres. El propio título es muy indicativo de la autoría y del contenido: Pronóstico experimentado y muy certero de Galeno. Nunca Galeno ni los restantes médicos griegos dijeron de un pronóstico que fuera 'verdadero',,$y$ menos aún 'muy verdadero'.

\section{Traducciones medievales}

No existen traducciones medievales del tratado (al sirio, al árabe o al latín)³.

\section{Los códices griegos ${ }^{4}$ y sus relaciones}

Conservan el texto griego tres manuscritos del siglo $\mathrm{XV}$ :

$\mathrm{P}=$ Parisinus gr. 22695, $\mathrm{M}=$ Mutinensis $\alpha . \mathrm{U} .9 .04$ (61 Puntoni $)^{6}, \mathrm{y} \mathrm{R}=$ Palatinus gr. $143^{7}$.

Los tres presentan faltas comunes y faltas propias.

Faltas comunes:

- Faltas por omisión:

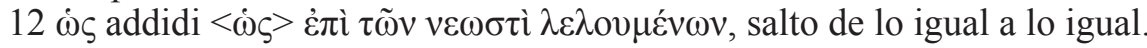

- Faltas por adición:

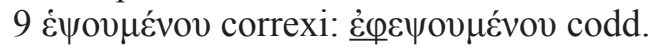

- Faltas por perseverancia:

2 ク̆ं

- Faltas por anticipación:

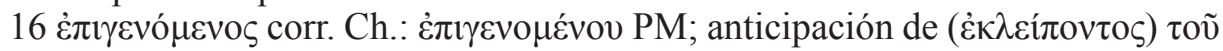

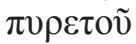

- Faltas por haplografía:

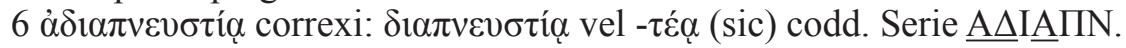

- Faltas por lectio facilior:

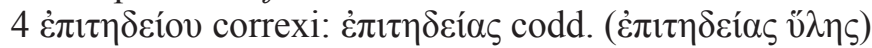

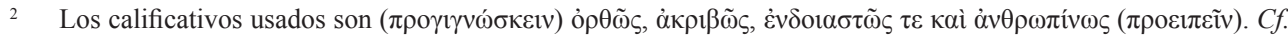
inter alia: Hipp. Prog. 25: 2.188.6 L. (= I 107.13 Kühlewein = Hipp. III 1: 77.4 Jouanna); Gal. In Hipp. Aph. III: 17b.570.1 K.; Hipp. Prorrh. 2.3: 9.10.24 L. (= Hipp. VIII 222.6 Potter). Muy rara vez se dice $\omega \varsigma \zeta \alpha \sigma \varphi \alpha \lambda \dot{\varepsilon} \alpha$,

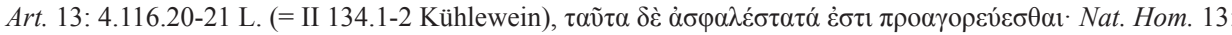
6.64.16 L. (= CMG I 1.3: 200.14 Jouanna). Por su parte, el adjetivo $\pi \alpha v \alpha \lambda \eta \theta \hat{\jmath} \varsigma$ solo se emplea junto a 'predecir' en contextos religiosos.

3 Véanse más adelante las traducciones latinas posteriores existentes.

$4 \quad C f$. Diels (1905: I 112).

$5 \quad C f$. Omont (1868: 0192). Véase también Touwaide (2016: 237).

$6 \quad C f$. Puntoni (1896: ref. 61). Véase ahora Touwaide (2016: 141).

$7 \quad C f$. Stevenson (1885: 74). Véase también Touwaide (2016: 279). 
$6 \pi \lambda \varepsilon \dot{\gamma} \mu \alpha$ corr. Ch: $\varphi \lambda \dot{\varepsilon} \gamma \mu \alpha$ codd.

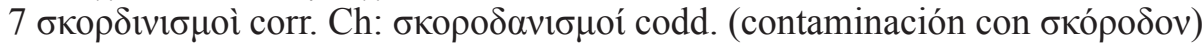

$14 \dot{\varepsilon} \vee \delta \varepsilon \kappa \alpha \tau \alpha i ̃ o l ~ c o r r . ~ C h: ~ \dot{~ \varepsilon ่ \nu \delta ́ ́ \kappa \alpha \tau o l ~ v e l ~ \varepsilon ́ v \delta \varepsilon ́ \kappa \alpha \tau o l ~ P M ~(y a ~ n o ~ R) ~}$

- Faltas fonéticas:

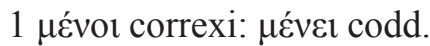

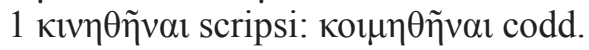

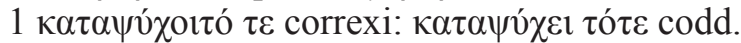

3 ๆ̈ $\pi \varepsilon \rho$ correxi: vं $\pi \dot{\varepsilon} \rho$ codd.

6 vं $\pi \rho \varphi \lambda \lambda \varepsilon \chi \theta \tilde{\eta}$ correxi: $\dot{\tau} \pi \rho \varphi \lambda \lambda \varepsilon \chi \theta \varepsilon i ́ \eta$ codd.

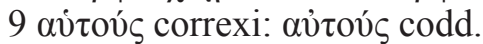

$10 \pi \rho \eta \sigma \theta \tilde{\omega} \sigma \iota v$ correxi: $\pi \rho ı \theta \tilde{\omega} \sigma \mathrm{v}$ codd.

$12 \pi \alpha \rho \alpha \pi \alpha i \omega \sigma \iota v$ scripsi: $\pi \alpha \rho \alpha \pi \varepsilon ́ \sigma \omega \sigma \iota v$ codd. Falta fonética y arreglo posterior

12 òvín correxi: àveín codd.

14 oi scripsi: cỉ codd.

Los manuscritos presentan faltas conjuntivas. Se trata de faltas fonéticas en su mayoría. Lo más importante es que no hay faltas de unciales, ni de minúsculas: la falta de las primeras indica texto del siglo IX o posterior, la de las segundas simplemente transmisión corta.

Faltas propias:

- Faltas propias de $\mathbf{P}$ contra $\mathbf{M}$ (ya no R):

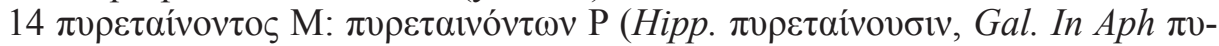

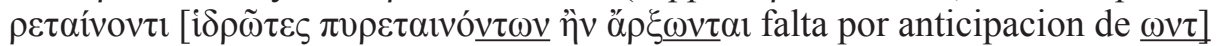
$15 \dot{\varepsilon} v \tau \alpha \tilde{v} \theta \alpha \mathrm{M}: \dot{\varepsilon} v \tau \alpha \tilde{\tau} \tau \alpha \operatorname{Par}$

- Faltas propias de M:

contra PR

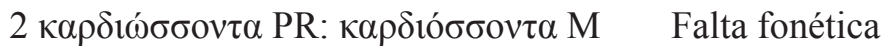

3 عi PR: $\dot{\eta} \mathrm{M} \quad$ Falta fonética

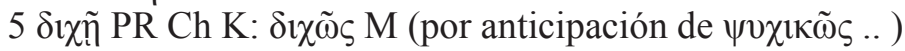

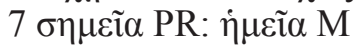

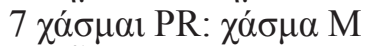

13 cĩval PR: ci̋̄n M

contra P (ya no hay R)

7 Eítovv $\dot{\alpha} \pi \circ \delta 1 \alpha \kappa \lambda \alpha \sigma \mu$ oí om. M, add. i.m. (salto de lo igual a lo igual: haplografía)

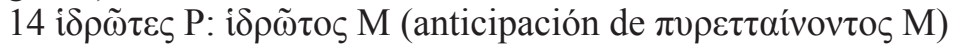

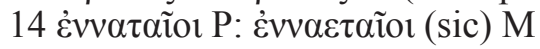

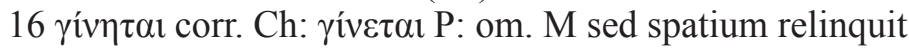

- Faltas propias de $\mathbf{R}$ contra PM

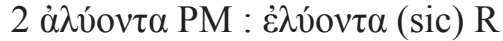

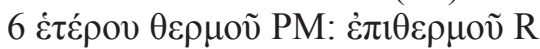

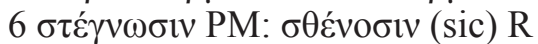

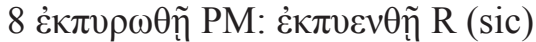

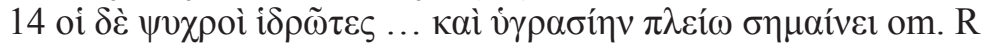

Tienen faltas conjuntivas: los tres manuscritos dependen de un ejemplar en minúsculas, en el que estaba escrito a continuación el De venae sectione. P acabó la 
copia del Praesagitio al final de su f. 117r, dejó en blanco el verso, y comenzó el De venae en $118 \mathrm{r}$.

El ejemplar que dio lugar a M y R no tenía una señal clara de explicit al final del Praesagitio, por lo que M y R lo copiaron a continuación, como si fuera el mismo tratado.

Por su parte, M y R comparten también faltas.

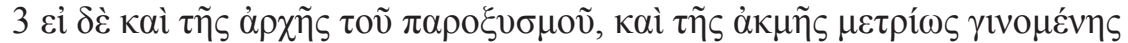
$\mu \varepsilon \tau \rho i ́ \omega \varsigma$ P: - $\alpha \varsigma$ MR puede haber surgido independientemente

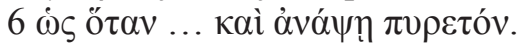

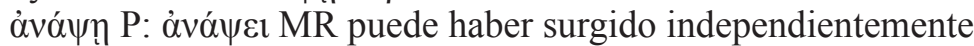

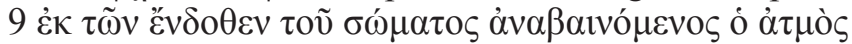

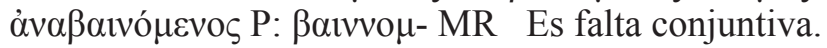

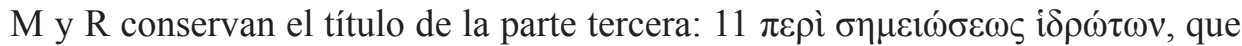
no aparece en P. Asimismo conservan la lección correcta frente a $\mathrm{P}$ en dos ocasiones:

- Faltas de P contra MR:

$4 \pi \rho \circ \sigma o \mu \imath \lambda i \alpha v$ MR : $\pi \rho \circ \sigma o \mu \eta \lambda i ́ \alpha v$ (sic) P

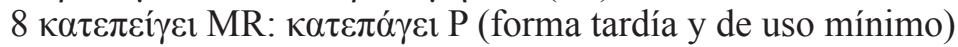

Creo poder afirmar en consecuencia que hay una copia perdida $\mu$ de la que proceden MR.

Las restantes faltas de grupo no son conjuntivas.

PM contra R] $6 \pi \rho \circ \sigma o \mu \imath \lambda \iota \alpha \nu$ R: $\pi \rho \circ \sigma o \mu \eta \lambda i ́ \alpha v$ (sic) PM.

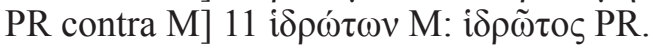

En cuanto a las faltas propias, $\mathrm{P}$ y $\mathrm{R}$ presentan pocas, mientras que $\mathrm{M}$ está escrito con mayor descuido. Por su parte $\mathrm{M}$ ha sido corregido:

$3 \tau$ $\chi_{\chi 01} \mathrm{M}^{\text {s.1. }}: \tau u ́ \chi \eta ~ P R M^{\text {i.1. }}$

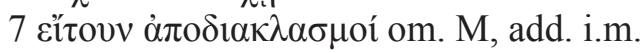

El copista de $\mathrm{P}$ es Demetrio Damilás ${ }^{8}$. El de M es Emmanuel Zacharides ${ }^{9}$.

\section{Stemma codicum}

post s. IX

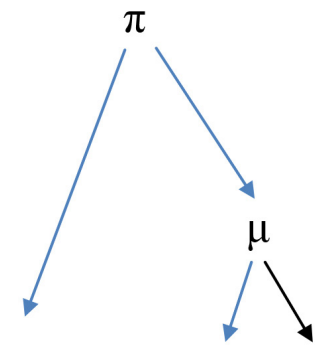

s. XV

P

$\mathrm{M} \quad \mathrm{R}$

8 Identificación de M. Sicherl (1991: 505, n. 50). Espécimen en http://webs.ucm.es/info/copistas/copista. html?num=181 (en 20 octubre 2020)

$9 \quad C f$. Vogel \& Gardthausen (1909: 126). 


\title{
5. Las ediciones del texto griego
}

El texto griego aparece por primera vez editado por René Chartier ${ }^{10}$ en 1639, y el editor se refiere a él con estas palabras: Est liber omnino malae notae ac spurius ${ }^{11}$. Como puede comprobarse, leyó el Parisinus gr. 2269:

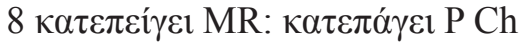

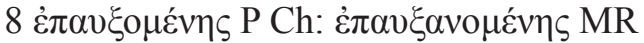 \\ $11 \pi \varepsilon \rho i ̀ ~ \sigma \eta \mu \varepsilon i \omega ́ \sigma \varepsilon \omega \varsigma$ i̇ $\rho \omega ́ \tau \omega \nu$ MR: om. P Ch

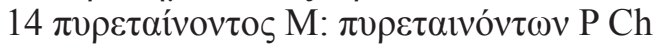

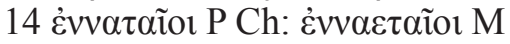

Hay que añadir que Chartier imprimió a continuación de Praesagitio el De venae sectione, en pp. 898-899, considerándolo un tratado diferente, tal como lo encontró en el Parisinus. Este editor dividió el texto en cuatro capítulos. Kühn, a su vez, imprimió la edición de Chartier con mínimas variaciones ${ }^{12}$.

\section{Las traducciones latinas}

Se han hecho sobre los manuscritos griegos existentes y por lo tanto no aportan nada salvo alguna posible corrección. Disponemos de la traducción renacentista de Giorgio Valla ${ }^{13}$, impresa en $1498^{14}$, y más tarde en la edición Juntina de 1565 , vol. 10 , f. $77 \mathrm{r}-\mathrm{v}$.

Inc. Quicunque praesciscere libuerit mortis non diem modo sed etiam horam Exp. longum namque morbum et humiditatis significat copiam

La traducción de Valla fue hecha sobre el Mutinense $\alpha$. U. 9. 04, que tenía en su Biblioteca ${ }^{15}$ :

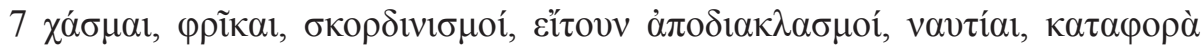
vँ $\pi$ vov

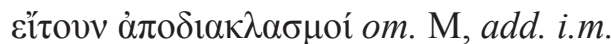

Valla : hiatus : horror : oscitatio.nausea.somnolentia

Se ha corregido $\mathrm{M}$ al margen más adelante. Valla no traduce el añadido.

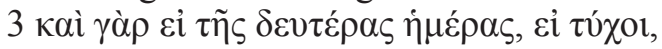
Ei prior] $\dot{\eta} \mathrm{M} \quad$ Valla: nam secunda die si contigat

A su vez, Charterius añadió a la edición del texto una traducción latina ${ }^{16}$ : Inc. Qui mortis non modo diem, sed et horam voluerim praenoscere Exp. Prorogatur enim morbus et copiosiorem significat humorem.

10 R. Charterius (1639: 895-897).

Véase su tomo VIII, p. 924. Cf. C. Petit (2012: 300).

Cf. Kühn (1830: 19.512.1 - 518.6).

13 Cf. nr. 138 de Durling (1961). Publicada en 1498, en Venecia por Simon Bevilaqua, y escrita en el último cuarto del siglo XV.

14 La digitalización es mala y no distingo la paginación, pero la traducción se encuentra en las imágenes 274-276. BNF (http://gallica.bnf.fr/ark:/12148/bpt6k58498k).

15 Cf. Heiberg (1896: 107-129).

16 VIII, 895-897. Es recogida por Kühn (vide infra). 


\section{Composición del tratado y fuentes: los loci similes}

Se trata de una agrupación de elementos diversos cuyo tema en común es la prognosis de muerte. Contiene tres apartados: 1. Pronóstico y paroxismo. 2. Las fiebres. 3. Los sudores y su significado.

En parte se aproxima a la literatura ¿por qué? o Quaestiones, como encontramos en los Problemata aristotélicos (atribuidos en su mayoría) ${ }^{17}$, o en los Problemata hipocráticos ${ }^{18}$, si bien las Quaestiones que ahora nos atañen han sido copiadas de otros textos.

LOCI SIMILES (remiten a los párrafos)

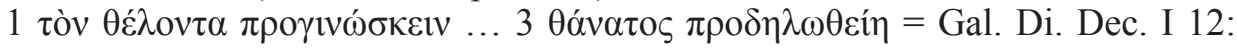
$9.831 .7-832.16 \mathrm{~K}$.

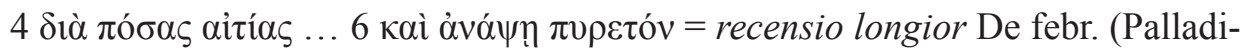
us) 9: I 111.1 -22 Ideler. $C f$. recensio brevior De febr. (Steph. -Theoph. $)^{19}$ 10.19. 24 - 30 Sicurus. Cf. Gal. Diff. Febr. 4: 7.282.10 - 283.1 K.

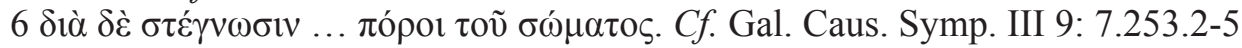

K. Cf. Gal. San. Tu. I 14: 6.76.4-5 K. (CMG V 4.2: I 14.9: 35.11-12 Koch)

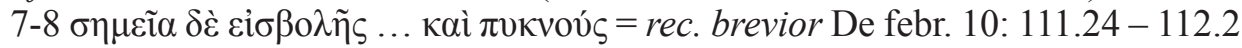
Ideler $=$ rec. longior De febr. 20: 23.17-30 Sicurus

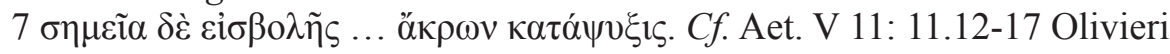

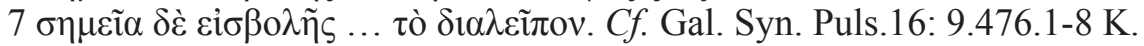

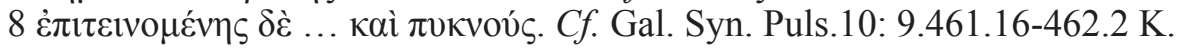

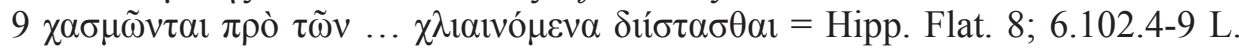
(Hipp. V 1: 113.11 - 114.3 Jouanna) = Pseudo-Gal. Caus. Aff.: 11.20-27 Helmreich

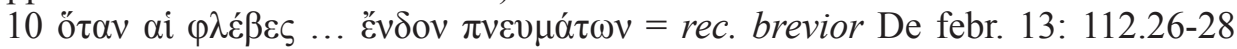
Ideler. $C f$. rec. longior De febr. 22; 25.1-5 Ideler. $C f$. Hipp. Flat. 8: 6.102.23 - 104.2 L. (Hipp V 1: 115.1-5 Jouanna)

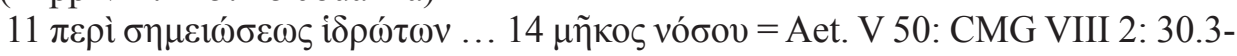
22 Olivieri

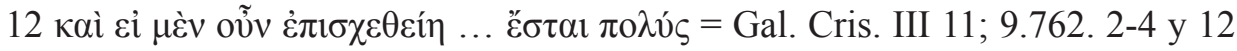
K. (207.23 - 208.2; 208.8-9 Alexanderson)

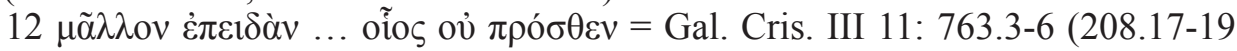
Alexanderson)

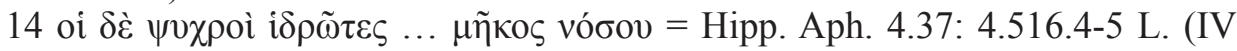
144.8-10 Jones) = Hipp. Cris. 26: 9.284.26-7 L. (9.14-6 Preiser = IX 286.24-5 Potter $)=$ Gal. Cris. I 19: 9.635.15-6 K. (122.20-1 Alexanderson $)=$ Gal. In Hipp. Aph. IV 37: 17b.715.9-10 K.= Theoph. Protosp. In Hipp. Aph. 37: II 408.7-9 Dietz = Steph. Athen. In Hipp. Aph. IV 35: 304.34-35 Westerink = Aet. V 50: 30.31-32 Olivieri. $C f$. Hipp. Progn. 6: 2.124.5-8 L. (86.14-16 Kühlewein I = Hipp. III 1: 16.5-7 Jouanna). $C f$. Steph. Athen. In Hipp. Progn. 11 (I 45): 120.6-8 Duffy. Cf. Steph. Athen. In Hipp. Aph. IV 34: 298.30-1 Westerink

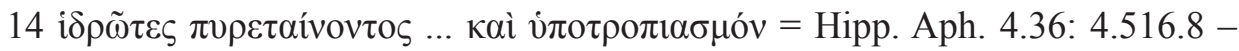
516.3 L. (IV 144.1-7 Jones) $=$ Hipp. Cris. 15: 9.280.23-6 L. (5.19 - 6.3 Preiser = IX

\footnotetext{
Bekker (1831).

Jouanna \& Guardasole (2017).

Véase Irmer (1973) y Monfort (en prensa $a$ y $b$ ).
} 
282.11-15 Potter $)=$ Gal. In Hipp. Aph. IV 36: 19b.711.7-13 K. = Theoph. Protosp. In Hipp. Aph. 36: II 406.22-9 Dietz = Steph. Athen. In Hipp. Aph. IV 34: 298.8-14 Westerink $=$ Steph. Athen. In Hipp. Aph. IV 34: 300.6-9 Westerink. Cf. Theoph. Protosp. In Hipp. Aph., 36: II 407.2-4 Dietz. Cf. Steph. Athen. In Hipp. Aph. IV 34: 298.23; 300.11-2; 300.20-1; 304.10-11 Westerink

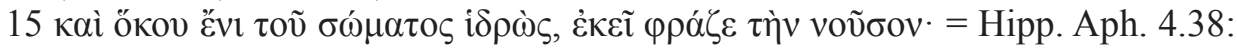
4. 516.6-7 L. (IV 144.11-2 Jones) = Gal. In Hipp. Aph. IV 38: 17B.717.13 K. = Theoph. Protosp. In Hipp. Aph. 38: II 409.5-6 Dietz = Steph. Athen. In Hipp. Aph. IV 36: 310.1-2 y 9 Westerink. Cf. Steph. Athen. In Hipp. Aph. IV 36: 310.10 Westerink

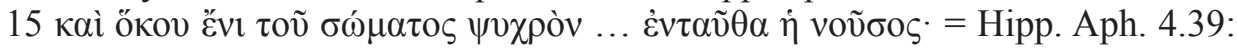
4.516.8-9 L. (= IV 144.13-4 Jones) = Hipp. Cris. 27: 9.284.27-8 L. (9.17-8 Preiser $=$ IX 288.1-2 Potter) = Gal. In Hipp. Aph. IV 39: 19b.718.7-8 K. = Theoph. Protosp. In Hipp. Aph. 39: II 409.16-17 Dietz = Steph. Athen. In Hipp. Aph. IV 37: 310.15-6 y 21-2 Westerink

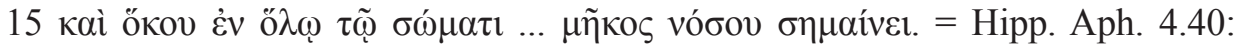
4.516.10-2 L. (= IV 144.15-7 Jones) = Hipp. Cris. 28: 9.284.29-31 L. (9.19-22 Preiser $=$ IX 288.3-6 Potter) = Gal. In Hipp. Aph. IV 40: 17b.718.12-719.2 K. = Gal. In Hipp. Aph. VII 62: 18a.175.9-11 K. y 176.3-6 K. = Theoph. Protosp. In Hipp. Aph. 40: II 409, 24-6 Dietz = II 543.5-7; 543, 11-13 Dietz = Steph. Athen. In Hipp. Aph. IV 37: 310.17-19 Westerink. $C f$. Steph. Athen. In Hipp. Aph. IV 37: 310.26-7 Westerink

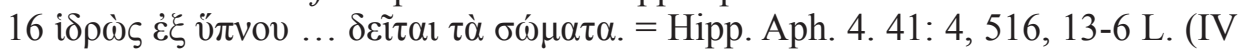
144.18-21 Jones) = Gal. In Hipp. Aph. IV 41: 17b.719.7-10 K.= Theoph. Protosp. In Hipp. Aph. 41: II 410.8-11 Dietz = Steph. Athen. In Hipp. Aph. IV 38: 312.6-9 Westerink. Cf. Steph. Athen. In Hipp. Aph. IV 38: 314.16-7 Westerink

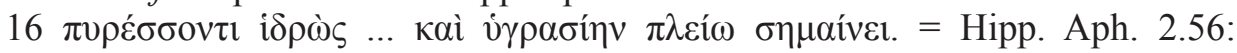
4.522.10-11 L. (IV 150.6-8 Jones) = Hipp. Cris. 29: 9, 286, 1-2 L. (10.1-3 Preiser = IX 288.7-8 Potter) = Gal. In Hipp. Aph. IV 56: 17b.734.9-11 K. = Theoph. Protosp. In Hipp. Aph. 56: II 419.17-9 Dietz = Steph. Athen. In Hipp. Aph. IV 53: 346.22-4 Westerink. Cf. Steph. Athen. In Hipp. Aph. IV 53: 348.5-6 Westerink

\section{Posible datación}

La fuente con datación segura más reciente es Aecio. No conocemos la fecha de la recensio brevior de De febribus, pero estas compilaciones podrían proceder de los siglos VII-IX ${ }^{20}$. Por otra parte, el texto aparece en los tres manuscritos seguido del De venae sectione, un tratado también muy tardío, compilado a partir de Oribasio y Pablo de Egina, lo que supone un post quem del siglo VII d.C. Es posible que ambos trataditos sean posteriores al siglo IX, aunque la copia de ambos en los tres manuscritos no lo hace indispensable.

Ahora bien, como no he observado faltas de mayúsculas, se tratará de un texto probablemente escrito en minúsculas. Por otra parte hay pocas divergencias entre los manuscritos, lo que indica que el común arquetipo dista poco de las copias existentes. Si el arquetipo y el original del autor fueran próximos, tendríamos una fecha reciente para el texto.

El hecho de que no existan traducciones medievales aboga, salvo error u omisión, por una fecha no muy lejana.

20 Véanse Grimm (2008: 30-35) y Wolska (1989: passim). 


\section{La lengua del tratado}

El inicio del libro con ö $\tau$, al que sigue una oración de infinitivo que depende de un $\chi \rho \eta ́$ posterior, es señal de un conocimiento incompleto de la sintaxis del griego antiguo, y apunta a una obra tardía. Hay muchos errores en el uso de subjuntivo y optativo, esperables asimismo en una obra tardía. Se utilizan mucho los aoristos pasivos:

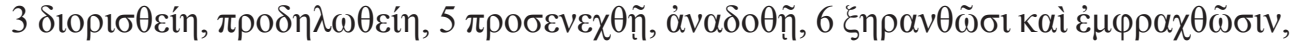

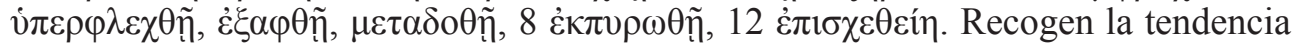
de Galeno a crear frases impersonales similares a la pasiva refleja del castellano.

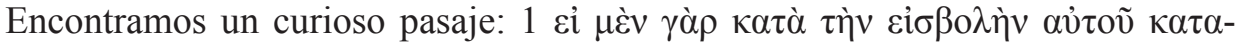

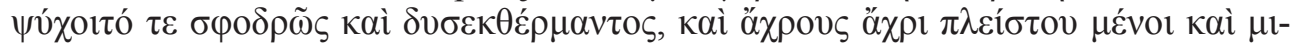

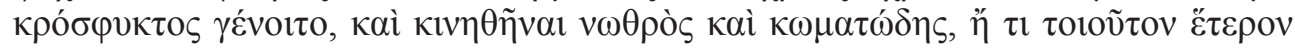

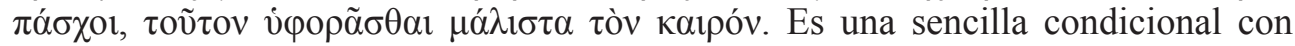

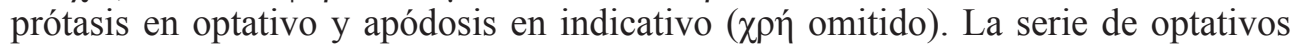

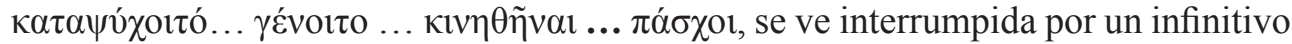

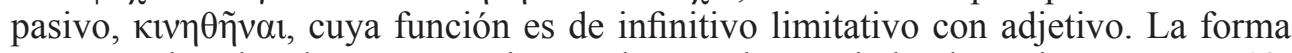
aparece tal en los doce manuscritos en los que he cotejado el pasaje Di. Dec. I 12: $9.831 .15 \mathrm{~K}$., y el editor del texto árabe, Cooper ${ }^{21}$, señala que éste se corresponde con

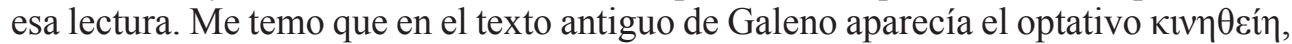
en justo paralelo a las restantes formas verbales de la condicional, y que una falta por

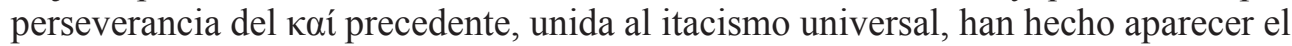
infinitivo.

Nos encontramos con algunos términos o formas excepcionales. De hecho el comparativo غ̇oiкó $\varepsilon \rho o \varsigma$ aparece solamente aquí según los datos del $T L G^{22}$, que tam-

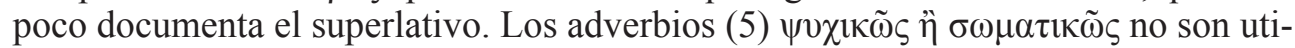
lizados por los médicos, y esta forma adverbial es tardía.

Hay una fuerte tendencia a emplear palabras largas a base de preverbios. Las he mantenido cuando aparecen en otros autores. En el caso de $\dot{\varphi} \varphi \varepsilon \psi o v \mu \varepsilon ́ v o v$ de los ma-

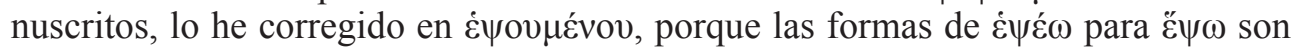

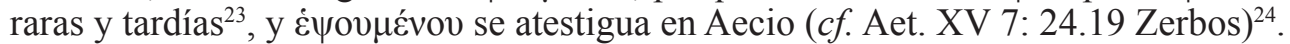

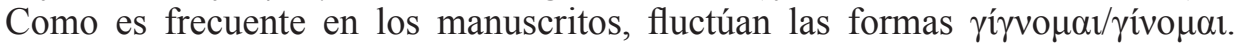
Como este texto no es de Galeno -en cuyo caso escribiríamos siempre las formas

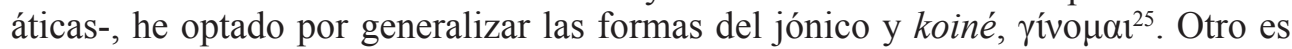
el caso en los textos tomados de Hipócrates -los referentes a los sudores-, donde aparecen las formas jónicas en estos manuscritos, como es habitual en todos los

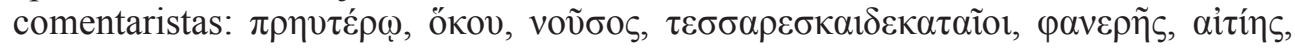
$\pi v \rho \varepsilon ́ \sigma \sigma o v \tau \imath$, v̛ $\rho \alpha \sigma i ́ \eta v$. Por ello las he mantenido. Para el condicional se usa siempre el contracto $\eta v$, muy habitual ya en escritores áticos.

En fin, en un texto de aluvión, que ha cogido pasajes de aquí y de allá, coexisten naturalmente formaciones diversas, como $\pi v \rho \varepsilon \tau \alpha i v o v \tau o \varsigma ~(14), \pi v \rho \varepsilon ́ \sigma \sigma o v \tau \imath ~(16), \pi v-$

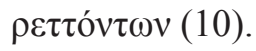

\footnotetext{
Cf. Cooper (2011: 208 and n. 510).

Leído en 20 octubre de 2020.

Véase Chantraine, $D E ́ L G$, s.v.

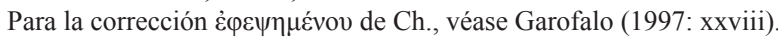

No se observa fluctuación en $\pi \rho \gamma \_$
} 


\section{Edición del texto}

\section{CONSPECTUS SIGLORUM}

M Mutinensis gr. $\alpha$. U. 9. 04 (61 Puntoni), s. xv

P Parisinus gr. 2269, s. xv

R Palatinus gr. 143, s. xv

Valla translatio latina a G. Valla facta, s. xv exeunte

$\mathrm{Ch}^{\text {lat }}$ translatio latina a R. Charterio facta, anno 1639

Ch editio graeca a Charterio facta, anno 1639

K editio graeca a C.G. Kühn facta, anno 1830

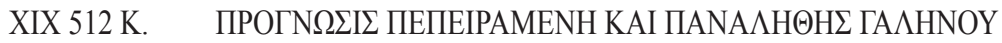

VIII $895 \mathrm{Ch}$.

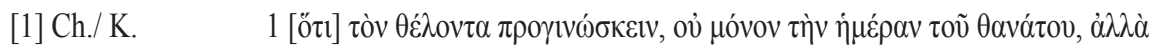

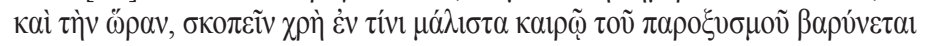

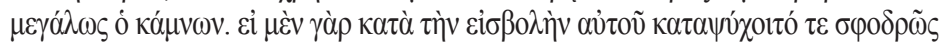

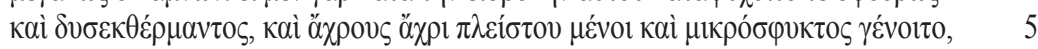

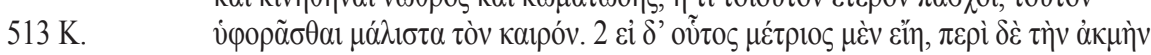

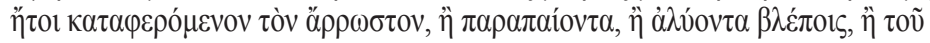

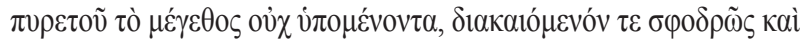

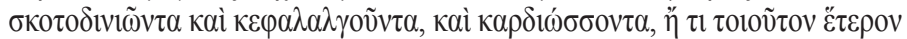

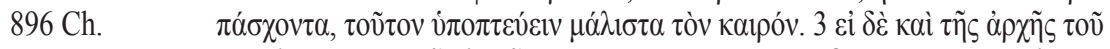

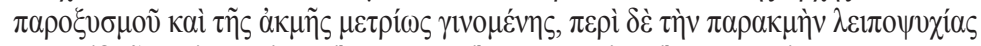

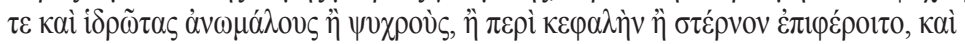

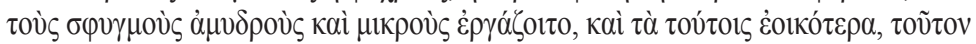
vं

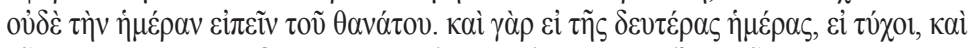

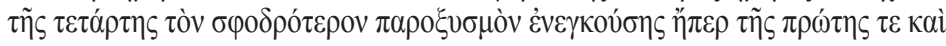

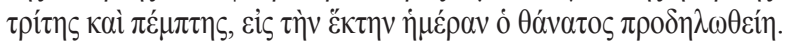

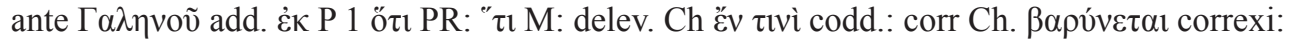

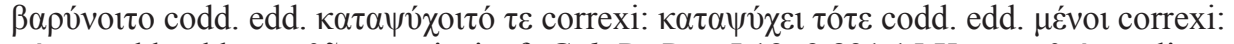

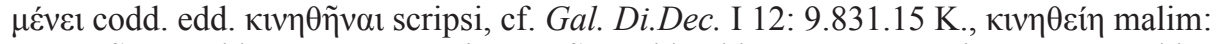

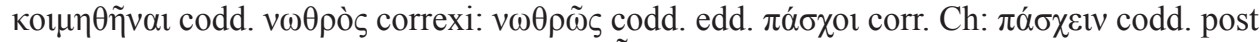

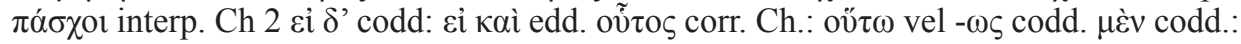

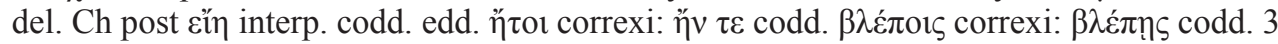

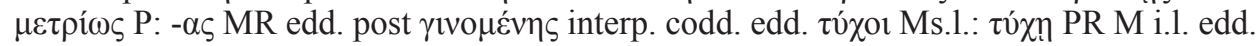

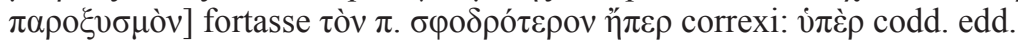




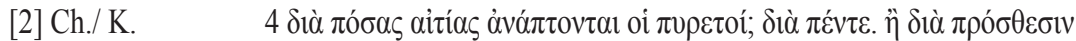

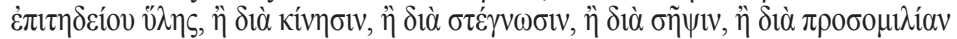

$514 \mathrm{~K}$.

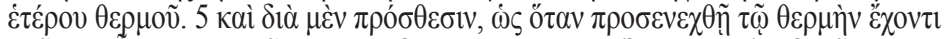

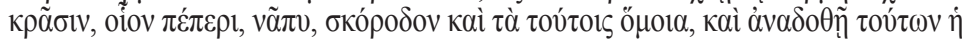

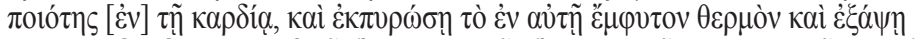

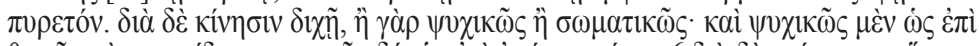

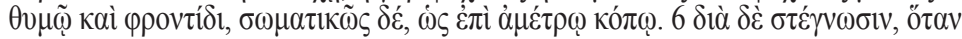

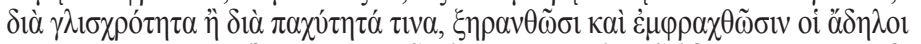

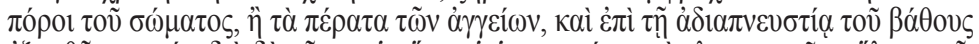

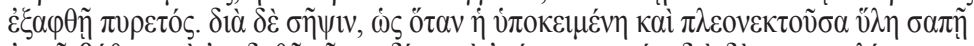

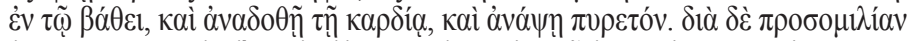

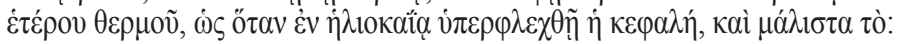

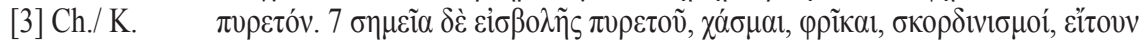

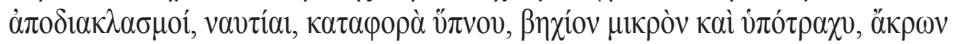

$515 \mathrm{~K}$.

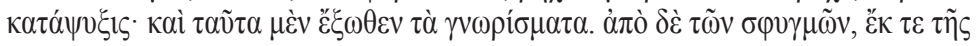

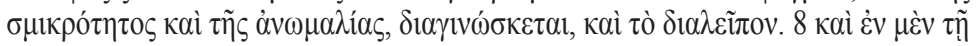

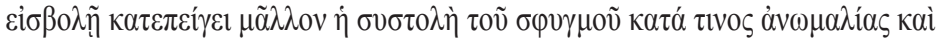

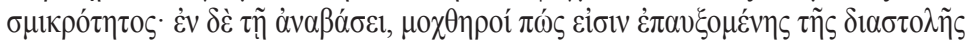

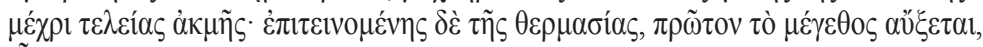

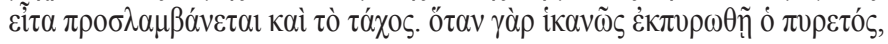

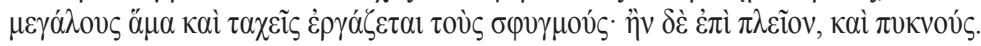

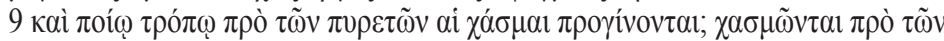

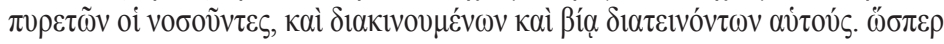

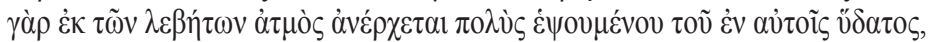

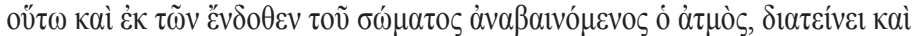

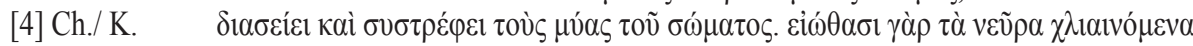

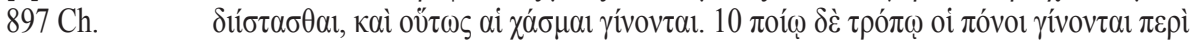
$516 \mathrm{~K}$.

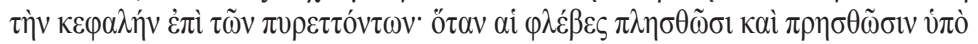

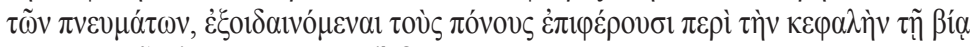

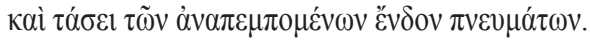

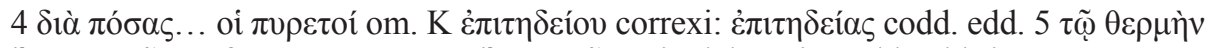

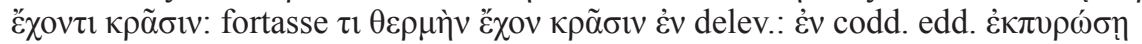

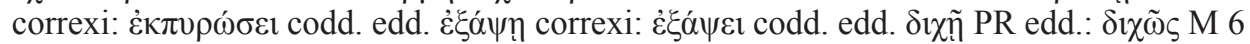

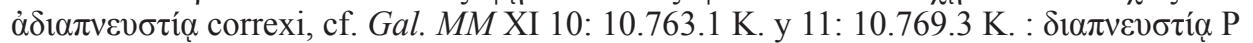

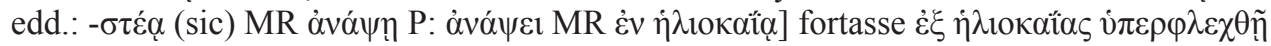

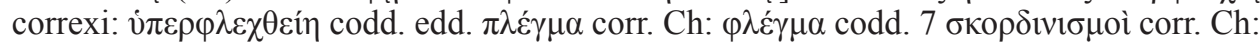

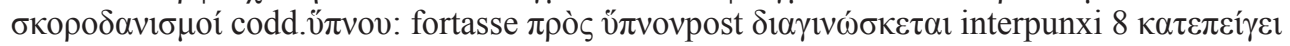

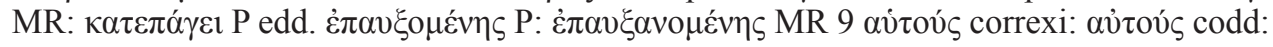

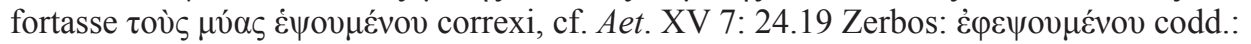

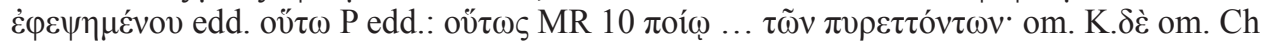
$\pi \rho \eta \sigma \theta \tilde{\omega} \sigma ı v$ correxi: $\pi \rho \imath \sigma \theta \tilde{\omega} \sigma ı v$ codd. edd. 


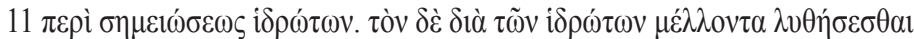

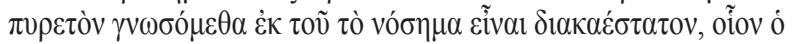

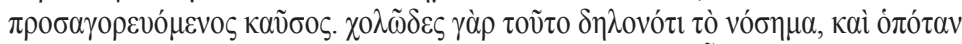

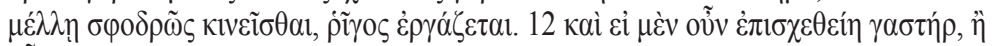

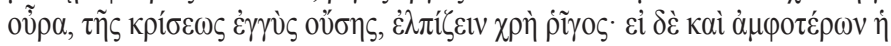

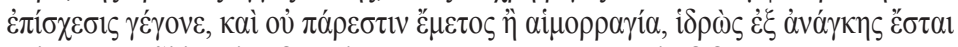

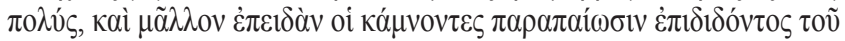

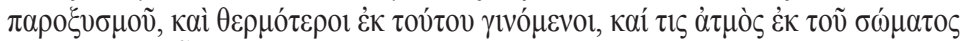

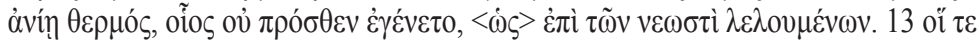

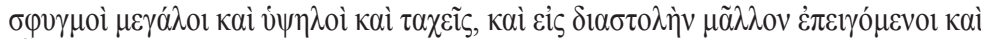

$517 \mathrm{~K}$.

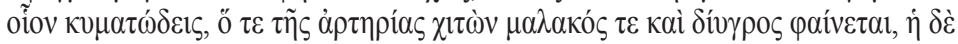

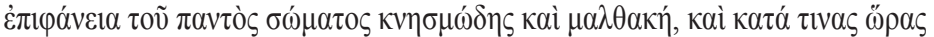

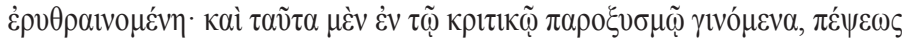

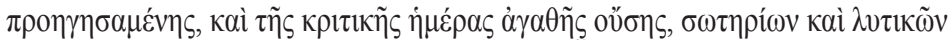

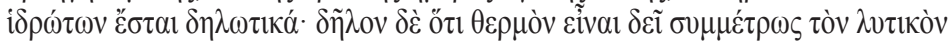

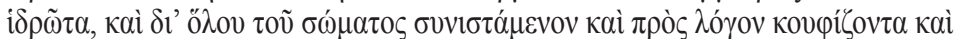

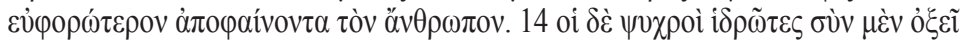

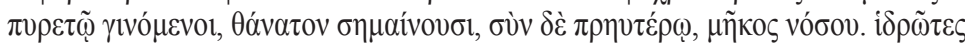

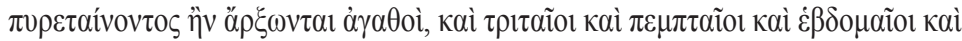

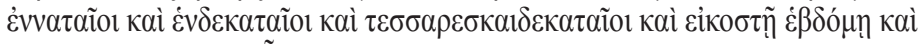

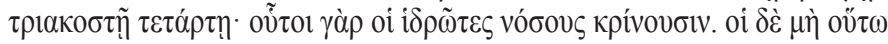

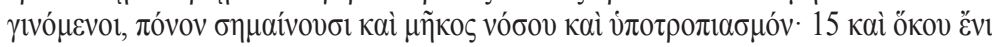

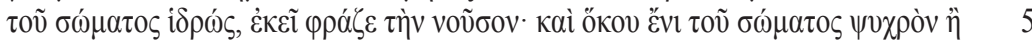

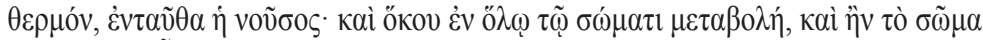

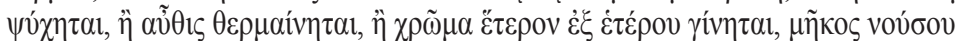

$518 \mathrm{~K}$.

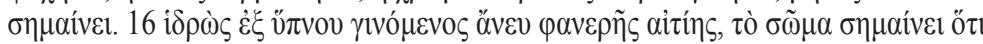

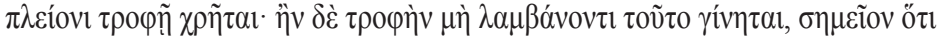

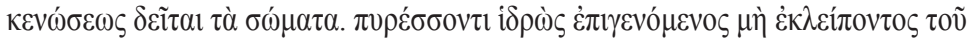

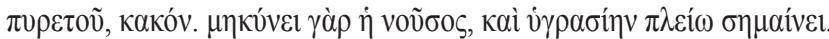

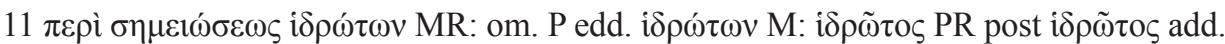

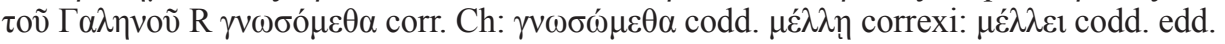

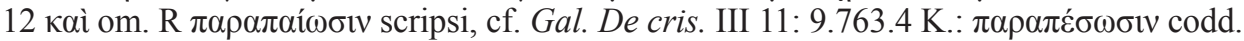

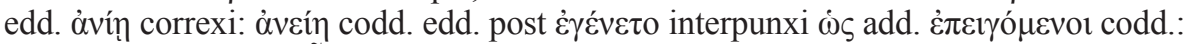

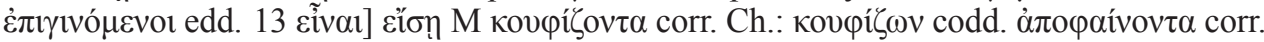

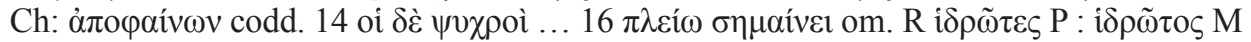
$\pi$

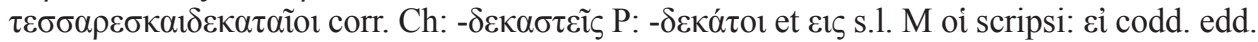

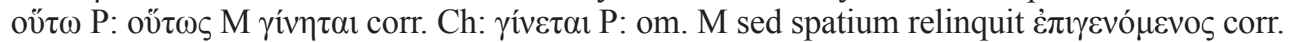

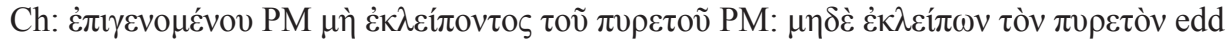

\section{Traducción}

\section{PRONÓSTICO EXPERIMENTADO Y MUY CERTERO DE GALENO.}

1. El que quiera pronosticar no solamente el día de la muerte, sino también la hora, es preciso que examine en qué momento precisamente del paroxismo está más apesadumbrado el enfermo. Pues si en el ataque de éste, él se sintiera muy frío y difícil de calentar, y se quedara completamente pálido y tuviera el pulso débil, y estuviera indolente y letárgico al moverse, o padeciera alguna otra cosa semejante, sospechar que ése es precisamente el momento (de la muerte). 2. Si éste (el pa- 
roxismo) fuera moderado, y en el momento culminante vieras al paciente inclinado (por sueño), o desvariando, o agitado, o no soportando la magnitud de la fiebre, o violentamente calentado, sufriendo mareos, dolor de cabeza, sufriendo cardialgia, o padeciendo alguna otra cosa de ese estilo, hay que suponer que ése es precisamente el momento (de la muerte). 3. Si no solo el comienzo del paroxismo, sino también el punto máximo, se produjeran moderadamente, $\mathrm{y}$, al descender, desvanecimientos y sudores anómalos o fríos, o en la cabeza o en el pecho, se añadieran, y se produjeran pulsos imperceptibles y cortos, y cosas más semejantes a ésas, suponer que ése es precisamente el momento (de la muerte). Y además, si eso se delimitara, ya no es nada difícil ni decir el día de la muerte. En efecto, si el segundo día, si se terciara, y el cuarto, trajeran el paroxismo más fuerte que el primero, el tercero y el quinto, al sexto día acaecería claramente la muerte.

4. ¿Por cuántas causas se generan las fiebres? Por cinco. O por añadidura de materia adecuada, o por movimiento, o por cierre, o por pudrición, o por proximidad de otra cosa caliente. 5. Por añadidura, como cuando se administre algo que tiene crasis caliente, como pimienta, mostaza, ajo y los semejantes a ésos, y se transmita de ésos la cualidad al corazón y se caliente en extremo el calor innato que hay en él y genere fiebre. Por movimiento, de dos maneras, o psíquica o somática. Psíquica, por una parte, por cólera o por preocupación, por otra parte, somática, como por un esfuerzo desmesurado. 6. Por cerramiento, cuando por viscosidad o por una cierta densidad se sequen y se bloqueen los poros invisibles del cuerpo, o los extremos de los vasos, y por la falta de transpiración de lo profundo se genere fiebre. Por pudrición, como cuando la materia subyacente, estando en demasía, se pudra en lo profundo, y se transmita al corazón, y avive la fiebre ${ }^{26}$. Por la asociación de otra cosa caliente, como cuando en una insolación se sobrecaliente la cabeza, y sobre todo la rete mirabile, y a través de las arterias se transmita al corazón y genere fiebre. 7. Señales del ataque de fiebre: bostezos, escalofríos, estiramientos de los miembros, o bien trastornos mentales, náuseas, ataque comatoso de sueño, tosecilla leve y un poco áspera, enfriamiento intenso de las extremidades, y ésas son señales desde fuera. Por otra parte, se diagnostica a partir de los pulsos, por su pequeñez e irregularidad, y por los intervalos. 8. Y en el ataque se acelera más la sístole del pulso con una cierta irregularidad y pequeñez. Por otra parte, en la subida son malignos de alguna manera (los pulsos) al aumentar la diástole hasta el punto máximo; al incrementarse el calor, en primer lugar aumenta la magnitud del pulso, después se le añade también la velocidad. Pues cuando la fiebre se caliente suficientemente, produce los pulsos grandes y rápidos. Y en caso de que vaya más allá, también frecuentes. 9. Y, ¿de qué manera antes de las fiebres se producen los bostezos? Bostezan antes de las fiebres los enfermos, al moverse y tensarse violentamente a sí mismos. Pues como desde los calderos sube mucho vapor al hervir el agua en ellos, así también desde dentro del cuerpo, al subir el vapor, tensa, agita y comprime los músculos del cuerpo. Pues suelen los tendones distenderse al calentarse, y así se producen los bostezos. 10. ¿De qué manera se producen los dolores de cabeza en los que tienen fiebre? Cuando las venas se llenen y se hinchen por obra de los aires, al hincharse provocan dolores en la cabeza, por la violencia y la presión de los aires interiores empujados hacia arriba.

11. Sobre lo que indican los sudores. Reconoceremos la fiebre que va a resolverse por medio de sudores a partir de ser la enfermedad (i.e. la fiebre) muy ardiente,

26 Para la fiebre sobre pudrición véase García Novo (2012: 218). 
como el llamado causón. Pues esa enfermedad es claramente biliosa, y cuando vaya a moverse (la bilis) fuertemente, se produce fiebre con escalofríos intensos ${ }^{27} .12$. Y si por una parte se retuviera el vientre, o la orina, estando cerca la crisis, hay que esperar fiebre con escalofríos intensos. Pero si se ha producido retención de ambos, y no aparece vómito o hemorragia, habrá necesariamente sudor en abundancia, y más cuando los enfermos, al ceder el paroxismo, deliren, y a partir de ahí calentándose más, también un cierto vapor caliente suba desde el cuerpo tal como antes no sucedió, como en los recién lavados. 13. Los pulsos grandes, elevados y rápidos, acelerándose más en la diástole, y por así decir como olas; la túnica de la arteria aparece blanda y muy húmeda, y la superficie de todo el cuerpo irritada y blanda, y a algunas horas enrojecida. Y esas cosas, produciéndose en el paroxismo coincidente con la crisis, habiendo acaecido antes la cocción, y siendo bueno el día de la crisis, indicarán sudores de recuperación y de remedio. Es evidente que la sudoración que remedia tiene que ser moderadamente caliente, produciéndose conjuntamente en el cuerpo completo, aliviando razonablemente y haciendo aparecer al individuo más sano. 14. Los sudores fríos que aparecen con fiebre aguda, señalan muerte; con fiebre más suave, enfermedad larga. Sudores del febricitante son buenos en caso de que aparezcan al comienzo, y también a los tres, cinco, siete, nueve, once, catorce, veintisiete y treinta y cuatro días: pues esos sudores traen la crisis de las enfermedades. En cambio los que no aparecen así, señalan sufrimiento, enfermedad larga y recidiva. 15. Y donde del cuerpo hay sudor, (tú) indica que allí está la enfermedad. Y donde del cuerpo haya una parte fría o caliente, allí está la enfermedad. Y donde ${ }^{28}$ en todo el cuerpo hay cambio, y en caso de que el cuerpo se enfríe, o de nuevo se caliente, o aparezca un color desde otro distinto, señala enfermedad larga. 16. Sudor produciéndose después del sueño, sin causa aparente, indica que el cuerpo se sirve de más comida. En caso de que eso suceda sin que coma más, es señal de que el cuerpo necesita una evacuación. Al febricitante que le sobreviene sudoración, sin que remita la fiebre, mala señal: pues la enfermedad se alarga e indica mayor humedad.

\section{Bibliografía}

Alexanderson, B. (1967), Galenos. ПEPI KPILEQN, Göteborg, Elander.

Bekker, I. (1831), Aristotelis opera, vol. 2, Berlin, Preussische Akademie der Wissenschaften (reimpr. Berlin 1960)

Chantraine, P. (1968-1980), = DÉLG, Dictionnaire étymologique de la langue grecque. Histoire des mots, Paris, Klincksieck (ampliada, 2009).

Charterius, R. (1639), Operum Hippocratis Coi, et Galeni Pergameni, medicorum omnium principum, t. VIII, Paris.

Cooper, G. M. (2011), Galen, De diebus decretoriis. A critical edition, with translation and commentary, of Ḥunayn Ibn Isḥāq, Kitāb Ayyām Al-Buhrān, Surrey-Burlington, Ashgate.

Diels, H. (1905-1907), Die Handschriften der antiken Ärzte. I. Teil. Hippokrates und Galenos, Abhandlungen der Kgl. Preuss. Akad. der Wissenschaften. Philos.-histor. Klasse, Berlin 1905. Suppl. Berlin 1907. http://cmg.bbaw.de/epubl/online/diels_02.html

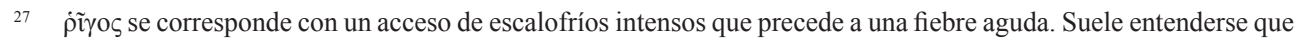
se refiere a una fiebre intermitente, como en la malaria. Cf. García Novo (2012: 236).

28 Debería decir "cuando" y no "donde", pero el texto ha mantenido el esquema de las oraciones anteriores. 
Dietz, F. R. (1834), 'Theophili et Damascii commentarii in Hippocratis aphorismos cum fragmentis et longioribus et brevioribus e Stephani Atheniensis philosophi, sive Meletii..', en: Scholia in Hippocratem et Galenum, ed., Vol. 2, Königsberg, Borntraeger (reimp. Amsterdam 1966): 236-544. https://www.biusante.parisdescartes.fr/histoire/medica/resultats/index.php?do=chapitre \& cote $=33148 \times 02$

Duffy, J. M. (1983), Stephani Philosophi In Hippocratis Prognosticum commentaria III, ed., CMG XI 1,2, Berlin, Akademie der Wissenschaften. http://cmg.bbaw.de/epubl/online/ cmg_11_01_02.php

Durling, R.J. (1961), «A Chronological Census of Renaissance Editions and Translations of Galen», Journal of the Warburg and Courtauld Institutes 24: 230-305.

García Novo, E. (2012), Galen. On the Anomalous Dyskrasia (De inaequali intemperie), ed., transl. and comment., Berlin, Logos Verlag.

Garofalo, I. (1997), Anonymi medici De morbis acutis et chroniis, ed., Leiden-New YorkKöln, Brill.

Grimm, I. (2008), Theophilos. Der Aufbau des Menschen, Kritische Edition des Textes mit Einleitung, Übersetzung und Kommentar, tesis doct., Munich, Ludwig-Maximilian Universität.

Heiberg, J.L. (1896), Beiträge zur Geschichte Georg Valla's und seiner Bibliothek, Leipzig, O. Harrassowitz.

Helmreich, G. (1910-1911), Handschriftliche Studien zu Galen. II, Gymn.Progr. Ansbach.

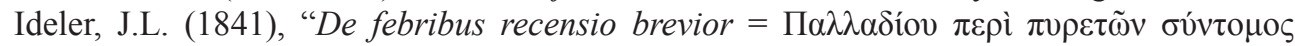
бúvouic", ed., en: Physici et medici Graeci minores, vol. 1, Berlin, Nabu: 107-20. https:// www.biusante.parisdescartes.fr/histoire/medica/resultats/index.php?do=chapitre \& cote $=33176 \times 01$

Irmer, D. (1973), «Bearbeitungen von Schriften des Iatrosophisten Palladius unter den Namen des Johannes, Stephanus und Theophilus», Medizinhist J. 8 (2-3): 179-181.

Jones, W.H.S. (1931), Hippocrates Vol. IV, Nature of Man. Regimen in Health. Humours. Aphorisms. Regimen 1-3. Dreams. Heracleitus: On the Universe, Cambridge, Mass. \& London, Harvard University Press.

Jouanna, J. (1985), Hippocrate. T. V 1, Des vents, De l'Art, ed., Paris, Belles Lettres.

Jouanna, J. (2002), Hippocratis De natura hominis, edidit, in linguam Francogallicam vertit, commentatus est, CMG I 1,3, Berlin, Akademie der Wissenschaften.

Jouanna, J. (2013), Hippocrate. T. III 1, Pronostique, ed., Paris, Belles Lettres.

Jouanna, J. \& Guardasole, A. (2017), Hippocrate. T. XVI, Problèmes hippocratiques, Paris, Belles Lettres.

Koch, C. \& Helmreich, G. (1923), Galeni De sanitate tuenda, De alimentorum facultatibus, De bonis malisque sucis, De victu attenuante, De ptisana, CMG V 4,2, Leipzig \& Berlin, Teubner. http://cmg.bbaw.de/epubl/online/cmg_05_04_02.php?p=67

Kühlewein, Hugo (1894), Hippocratis Opera quae feruntur omnia, I, Leipzig, Teubner. http://cmg.bbaw.de/epubl/online/wa_hippocrates_op_1.php

Kühn, K.G. (1821-1833), Claudii Galeni Opera Omnia, Leipzig, C. Cnobloch. https://www. biusante.parisdescartes.fr/histoire/medica/resultats/index.php?fille $=$ o\&cotemere $=45674$

Littré, É. (1839-1861), Oeuvres complètes d'Hippocrate, Paris, J.B. Baillière. https://www. biusante.parisdescartes.fr/histoire/medica/resultats/index.php?fille $=$ o\&cotemere $=34859$

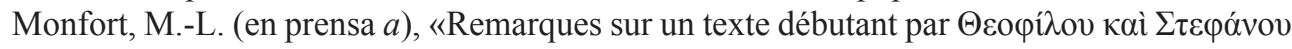

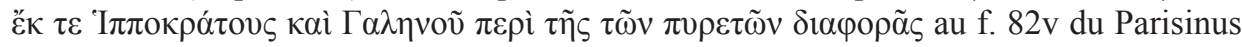
gr. 2219», en Un corpus médical problématique. Les traités attribués à Théophile Protospathaire (VIIe-IXe s.) et la relecture chrétienne des enseignements hippocratiques et galéniques, Colloque international organisé par Alessia Guarsasole et Caroline Magdelaine, Paris, Université de Paris-Sorbonne, 27-28 novembre 2014. 
Monfort, M.-L. (en prensa $b$ ), «Les compilations du De differentiis febrium de Galien dans les deux versions de l'Anonyme Sur les fièvres», en Internationale medizinhistorische Tagung Sammlung und Fragmentierung: Medizinische Kompilationen des Morgen- und Abendlands und ihre Quellen, Charité Universitätsmedizin, Berlin, 3009 - 02102017 (organisé par Mathias Witt).

Olivieri, A. (1950), Aetii Amideni Libri medicinales V-VIII, ed., CMG VIII 2, Berlin, Akademie der Wissenschaften. http://cmg.bbaw.de/epubl/online/cmg_08_02.php

Omont, H (1886-1898), Inventaire sommaire des manuscrits grecs de la Bibliothèque nationale et des autres bibliothèques de Paris et des Départements, Paris, Belles Lettres. https://gallica.bnf.fr/ark:/12148/bpt6k2091353.image

Petit, C. (2012), «René Chartier (1572-1654) et l'authenticité des traités Galéniques», en G. Cobolet et alii (eds), René Chartier (1572-1654) éditeur et traducteur d'Hippocrate et Galien, Paris, De Boccard: 287-300.

Potter, P. (1995), Hippocrates Vol. VIII, Places in Man. Glands. Fleshes. Prorrhetic 1-2. Physician. Use of Liquids. Ulcers. Haemorrhoids and Fistulas, Cambridge, Mass. \& London, Harvard University Press.

Potter, P. (2010), Hippocrates Vol. IX. Coan Prenotions. Anatomical and Minor Clinical Writings (Crises and Critical Days), Cambridge, Mass. \& London, Harvard University Press.

Preiser, G. (1957), Die hippokratischen Schriften "De iudicationibus" und "De diebus iudicatoriis", tesis doct., Kiel.

Puntoni, V. (1896), «Indice dei codici greci della Biblioteca Estense di Modena», Stud.Ital. 4: 379-536. https://pinakes.irht.cnrs.fr/notices/bibliographie/6JFRTFUX/

Sicherl, M. (1991), «Handschriftenforschung und Philologie», en D. Harlfinger \& G. Prato (eds.), Paleografia e codicologia greca. Atti del II Colloquio internazionale. BerlinoWolfenbüttel (17-21 ottobre 1983), 2 vols., Alessandria, Edizioni dell'Orso: 485-508.

Sicurus, D. (1862), "De febribus recensio longior" = Theophili et Stephani Atheniensis de febrium differentia ex Hippocrate et Galeno, ed., Firenze, Bencinii (reimp. Amsterdam 1963).

Stevenson, E. (1885), Codices manuscripti Palatini Graeci Bibliothecae Vaticanae, Roma, Ex Typographeo Vaticano. https://archive.org/details/codicesmanuscrip00stevuoft

Touwaide, A. (2016), A Census of Greek medical manuscripts from Byzantium to the Rennaisance, Londres \& New York, Routledge.

Valla, Giorgio (1498), “Galeni De Praesagitura”, en: Collectio. Nicephori Logica. De expedita ratione argumentandi. Euclidis Quartus decimus Elementorum ... Giorgio Valla Placentino Interprete, Venezia, Simon Bevilaqua. https://gallica.bnf.fr/ark:/12148/bpt6k58498k.r=Giorgio\%20Valla?rk=85837;2

Valla, Giorgio (1565), “Galeni De Praesagitura”, en: Galeni omnia quae extant opera, vol. 10. Ascripti libri, Venezia, apud Iuntas, 77 r-v. https://archive.org/details/galeniomniaquaee67gale

Vogel, M. \& Gardthausen, V. (1909), Die griechischen Schreiber des Mittelalters und der Renaissance, Berlin, Otto Harrasowitz. https://archive.org/details/diegriechischens00vogeuoft

Westerink, L. G. (1992), Stephani Atheniensis In Hippocratis Aphorismos commentaria IIIIV, CMG XI 1,3,2, Berlin, Akademie der Wissenschaften. http://cmg.bbaw.de/epubl/online/cmg_11_01_03_02.php

Withington, E. T. (1948), Hippocrates. Vol. III. On Fractures, On Joints, Instruments of Reduction, London \& Cambridge, Mass., Harvard University Press https://www.loebclassics.com/view/hippocrates_cos-fractures_joints_mochlicon_fractures/1928/pb_ LCL149.95.xml?rskey $=$ GEZ1SR\&result $=1$ 
Wolska-Conus, W. (1989), «Stephanos d'Athènes et Stephanos d'Alexandrie: Essai d'identification et de biographie», REB $47: 5-89$.

Zervos, S. (1909), Aetii Amideni Liber medicinalis XV, ed., Athena 21: 3-144. http://cmg. bbaw.de/epubl/online/wa_Athena_23_1911.php 